\title{
Candida krusei
}

National Cancer Institute

\section{Source}

National Cancer Institute. Candida krusei. NCI Thesaurus. Code C77173.

A species of budding yeast in the phylum Ascomycota. 